\title{
Using convolutional neural networks for hygrothermal predictions to extrapolate to other external climates
}

\author{
Astrid Tijskens ${ }^{1, *}$, Hans Janssen ${ }^{1}$, and Staf Roels ${ }^{1}$ \\ ${ }^{1}$ KU Leuven, Department of Civil Engineering, Building Physics Section, Kasteelpark Arenberg 40 Bus 2447, 3001 Heverlee, Belgium
}

\begin{abstract}
When simulating the hygrothermal behaviour of a building component, many uncertainties are involved (e.g. exterior and interior climates, material properties, configuration geometry). In contrast to a deterministic assessment, a probabilistic analysis enables including these uncertainties, and thus allows a more reliable assessment of the hygrothermal performance. This easily involves thousands of simulations, which easily becomes computationally inhibitive. To overcome this time-efficiency issue, a convolutional neural network, a type of metamodel mimicking the original model with a strongly reduced calculation time, can replace the hygrothermal model. This was proven in a previous study for a massive masonry wall, where variability of exterior and interior climate, brick material properties and wall geometry was included. However, the question rises whether it is possible to train the network on a limited number of climates, and afterwards use the network to predict accurately for other climates as well. This paper thus focuses on this aspect, and results show that, as long as the range of the new climate data falls within the range of the climate data the network was trained on, the network is able to predict accurately for new climates as well.
\end{abstract}

\section{Introduction}

Evaluating the hygrothermal performance of a building component in a probabilistic framework [1,2] allows considering many uncertainties, such as the exterior and interior climate, the material properties, or even the configuration geometry. It thus allows a more reliable assessment of the hygrothermal performance and the potential moisture damages, in contrast to a deterministic assessment. Often the Monte Carlo approach [3] is used, where the uncertain input parameters' distributions are sampled several times and a deterministic simulation is executed for each sampled parameter combination. However, this approach easily requires thousands of simulations, and thus becomes computationally inhibitive. By replacing the original hygrothermal model by a much simpler and faster metamodel, which mimics the original model, the calculation time can be reduced significantly. In the context of hygrothermal performance prediction, dynamic metamodels have a powerful advantage over static metamodels. Static metamodels are developed for a specific single-valued performance indicator (e.g. the total heat loss or the maximal mould growth index), whereas dynamic metamodels aim to predict time series (temperature, relative humidity, moisture content, ...). The latter thus provide a more flexible approach, as predicting the hygrothermal time series allows post-processing by any desired damage prediction model (e.g. the VTT mould growth index), but also provides information over the whole evaluation period. Nonetheless, developing a dynamic metamodel is also more difficult, as the metamodel must be able to capture the complex and time dependent pattern between input and output time series, and not all metamodelling strategies are suited for time series prediction.

In previous studies $[4,5]$, the authors demonstrated that convolutional neural networks (CNN) are well suited to predict the dynamic hygrothermal response of a building component. In [5], a network architecture is developed and optimized to predict the hygrothermal behaviour of a massive masonry wall, in a probabilistic framework including uncertainties such as interior and exterior climate, brick wall thickness and brick type. The network was trained on a small dataset obtained by sampling the probabilistic input parameters, and was found to accurately predict the hygrothermal performance of the massive masonry wall. Yet, this network was trained to predict for specific climates and a single wall type only. The question rises whether it is possible to train such a network on a dataset with a limited number of cases, and afterwards use it to extrapolate and accurately predict the hygrothermal performance of other wall types, materials or exterior climates. This would significantly increase the metamodel's power, as a single metamodel could be employed for a wide variety of cases without the need to increase the training dataset. Therefore, this paper is a first explorative study, which focuses on the case of a massive masonry wall and the possibility to use the CNN to extrapolate to other climates after training on a limited dataset.

\footnotetext{
* Corresponding author: astrid.tijskens $@$,kuleuven.be
} 


\section{Calculation object}

A 1D massive masonry wall cross section is used as calculation object. The masonry wall is simplified to an isotropic brick layer [6], no mortar joints and construction details such as corners or embedded beams are modelled. Figure 1 shows a schematic representation of the considered 2D building component and the modelled 1D mesh. All characteristics and boundary conditions that are expected to significantly influence the hygrothermal performance of the 1D wall are considered probabilistic (Table 1). Variability in climatic conditions is included by using different years of climate data [7], variability in the wall conditions is incorporated via uniform distributions of the wall orientation, solar absorption and exposure to wind-driven rain. The wind-driven rain load is calculated by using the catch ratio as described in [8]. The catch ratio relates the wind-driven rain (WDR) intensity on a facade to the unobstructed horizontal rainfall intensity and is a function of the reference wind speed and the horizontal rainfall intensity for a given position on the building facade and wind direction. In this model, variability in wall position and potential shelters, trees or surrounding buildings are reckoned with by the exposure factor. Additionally, the transiency and variation of the wind speed is taken into account in the convective heat transfer coefficient, via equation (1) (EN ISO 06946) where $h_{0}=$ $4 \mathrm{~W} / \mathrm{m}^{2} \mathrm{~K}, k_{e}=1$ and $v$ is the wind speed.

$$
h_{c}=h_{0}+k_{s} \cdot v^{k_{e}}
$$

The exterior moisture transfer coefficient is related to the exterior heat transfer coefficient through the Lewis relation. The interior climate is calculated according to EN 15026 [9] and variability in building use is included by using two different humidity loads A and B. Finally, variability in materials characteristics and geometry is added by including three different brick types as well as a uniform distribution of the wall thickness.

Table 1. Probabilistic input parameters and distributions.

\begin{tabular}{|c|c|}
\hline Parameter & Distribution \\
\hline Exterior climate start year & $\mathrm{D}(2020 ; 2047)$ \\
\hline $\begin{array}{l}\text { Wall orientation [degrees } \\
\text { from north] }\end{array}$ & $\mathrm{U}(0 ; 360)$ \\
\hline Solar absorption [-] & $\mathrm{U}(0.4 ; 0.8)$ \\
\hline $\begin{array}{l}\text { Ext. heat transfer coefficient } \\
\text { slope } k_{S}\left[\mathrm{~J} / \mathrm{m}^{3} \mathrm{~K}\right]\end{array}$ & $\mathrm{U}(1 ; 8)$ \\
\hline WDR exposure factor & $\mathrm{U}(0 ; 2)$ \\
\hline Brick wall thickness [m] & $\mathrm{U}(0.2 ; 0.5)$ \\
\hline Brick material & $\begin{array}{c}\text { D(Brick1; Brick2; } \\
\text { Brick3) }\end{array}$ \\
\hline
\end{tabular}

The remaining parameters are all variables either with small variations or of less importance for the current study of a 1D wall. Therefore, these boundary conditions are assumed deterministically.
The hygrothermal performance of the masonry wall is evaluated at three positions, as indicated on Figure 1: At the exterior surface, frost damage is assessed by the number of moist freeze-thaw cycles. At $5 \mathrm{~cm}$ from the interior surface, decay of embedded wooden floors is assessed using the VTT wood decay model and at the interior surface, mould growth is assessed using the updated VTT mould growth model. To estimate whether these damage patterns will occur, the temperature $(\mathrm{T})$, relative humidity $(\mathrm{RH})$ and saturation degree are monitored at the associated positions for a period of 4 years, at an hourly frequency.

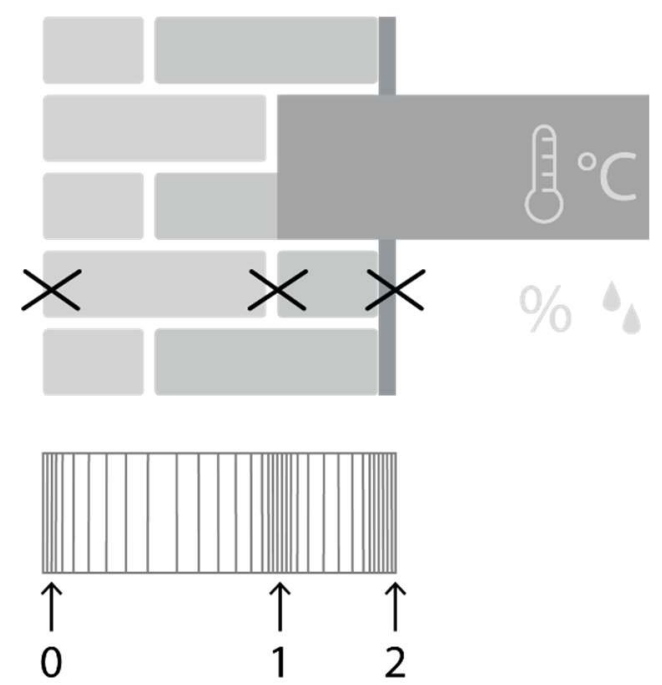

Fig. 1. A schematic representation of the $2 \mathrm{D}$ building component (top) and the modelled 1D mesh (bottom), with indication at which positions the hygrothermal performance is monitored.

\section{Convolutional neural network}

The architecture of the CNN is described in detail in [5]. The network consists of stacked residual blocks with causal dilated convolutions. Causal convolutions ensure that the output of the filter does not depend on future inputs. In a dilated convolution, the filter is applied over an area larger than its length by skipping input values with a certain step. Stacked dilated convolutions enable the network to access information over a large time span - the receptive field - with just a few layers, while preserving the input sequence length throughout the network as well as maintaining computational efficiency. The dilation factor is exponentially increased for every layer, allowing the receptive field to grow exponentially with network depth. In this paper, three stacks of three residual blocks are used. In each convolutional layer, 256 filters are used with a length of 10 .

The network is trained to predict the hygrothermal time series as requested for the damage prediction models. The inputs are pre-processed to facilitate learning. The climate time series exterior temperature, exterior relative humidity, wind-driven rain load, short-wave radiation, interior temperature and interior relative humidity are the main input. Longwave radiation is included in the hygrothermal simulations, but discarded as network input 
due to its limited predictive power. The scalar parameters 'wall orientation', 'exterior heat transfer coefficient slope', 'solar absorption' and 'rain exposure' are integrated in the exterior climate time series but also preserved as separate scalar input, to condition the network on different situations. The categorical parameters 'start year' and 'interior humidity load' are incorporated into the climate time series. The categorical parameter 'brick type' is replaced by scalar parameters of the characteristics (dry thermal conductivity, dry vapour resistance factor, capillary absorption coefficient, capillary moisture content, saturation moisture content). This simplifies the network architecture, and allows more flexibility on using brick types with differing characteristics. This results in 6 time series inputs (exterior temperature, exterior relative humidity, winddriven rain load, short-wave radiation, interior temperature, interior relative humidity) and 10 scalar inputs (exterior heat transfer coefficient slope (eq. (1)), rain exposure factor, solar absorption, wall orientation, brick wall thickness and the 5 brick characteristics).

Before presenting the input and output data to the neural network, all data are standardised (zero mean, unit variance). This ensures that all features are on the same scale, which allows weighting all features equally in their representation. Standardising the output data ensures that errors are penalised equally for all targets. To overcome weight initialisation differences, the network is trained five times on the same dataset. The accuracy of these five networks is evaluated by three indicators: the root mean square error (RMSE), mean absolute error (MAE), and coefficient of determination (R2), formulated as follows:

$$
\begin{gathered}
R M S E=\sqrt{\sum \frac{(y-\hat{y})^{2}}{T}} \\
M A E=\sum \frac{y-\bar{y}}{T} \\
R 2=1-\frac{\sum(y-\hat{y})^{2}}{\sum(y-\bar{y})^{2}}
\end{gathered}
$$

where $y$ is the true output, $\hat{y}$ is the predicted output, $\bar{y}$ is the mean of the true output and $T$ is the total number of time steps. To remove scale differences between the different outputs, these indicators are calculated on the standardised outputs. Additionally, the training time of the neural network is evaluated.

To investigate the possibility to train the network on a limited set of exterior climates and afterwards use it to predict the hygrothermal performances for other climates as well, the CNN is trained on two different datasets, each containing different climates. The first training dataset includes four Belgian climates only (Gent, Gaasbeek, Oostende, St Hubert). The network trained on this dataset, is called CNN-BE. The second training dataset includes four Swedish climates (Stockholm, Östersund, Borlänge, Lund) and four Italian climates (Ancona, Naples, Milan, Crotone). The network trained on this dataset, is called CNN-SE-IT. Both training datasets contain 768 samples, regardless of the number of climates that were included. Note that in case of the SE-IT dataset, there are fewer samples per climate, compared to the BE dataset. To ensure the networks have good performance for the climates they were trained on, they are evaluated on a validation dataset containing 256 samples that differ from the respective training dataset but include the same climates. All datasets are obtained by sampling the input parameters described in section 2.1, using a Sobol sampling scheme [10], and simulating the deterministic HAM model once for each sampled input parameter combination. The number of samples in each dataset was chosen based on previous experiments, where this amount was found sufficient. However, it is possible that a larger or smaller training dataset might result in higher or equally satisfying prediction performance respectively.

Next, the networks' performance can be tested when used to extrapolate to climates that were not included in their respective training dataset. For this purpose, three test datasets are created, one for each climatic country (BE, SE, IT), each containing 256 samples.
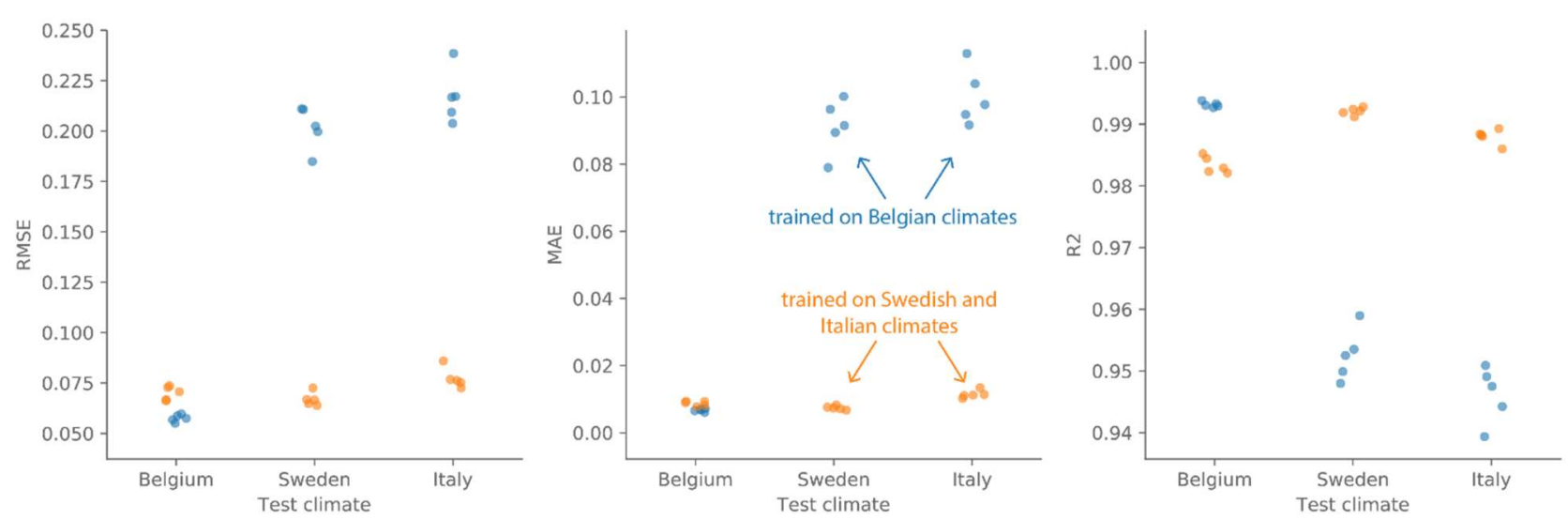

Fig. 2. The networks' prediction performance on the test datasets indicates that CNN-BE (blue) is not able to predict for the Swedish and Italian climates, whereas CNN-SE-IT (orange) can predict for the Belgian climate. 


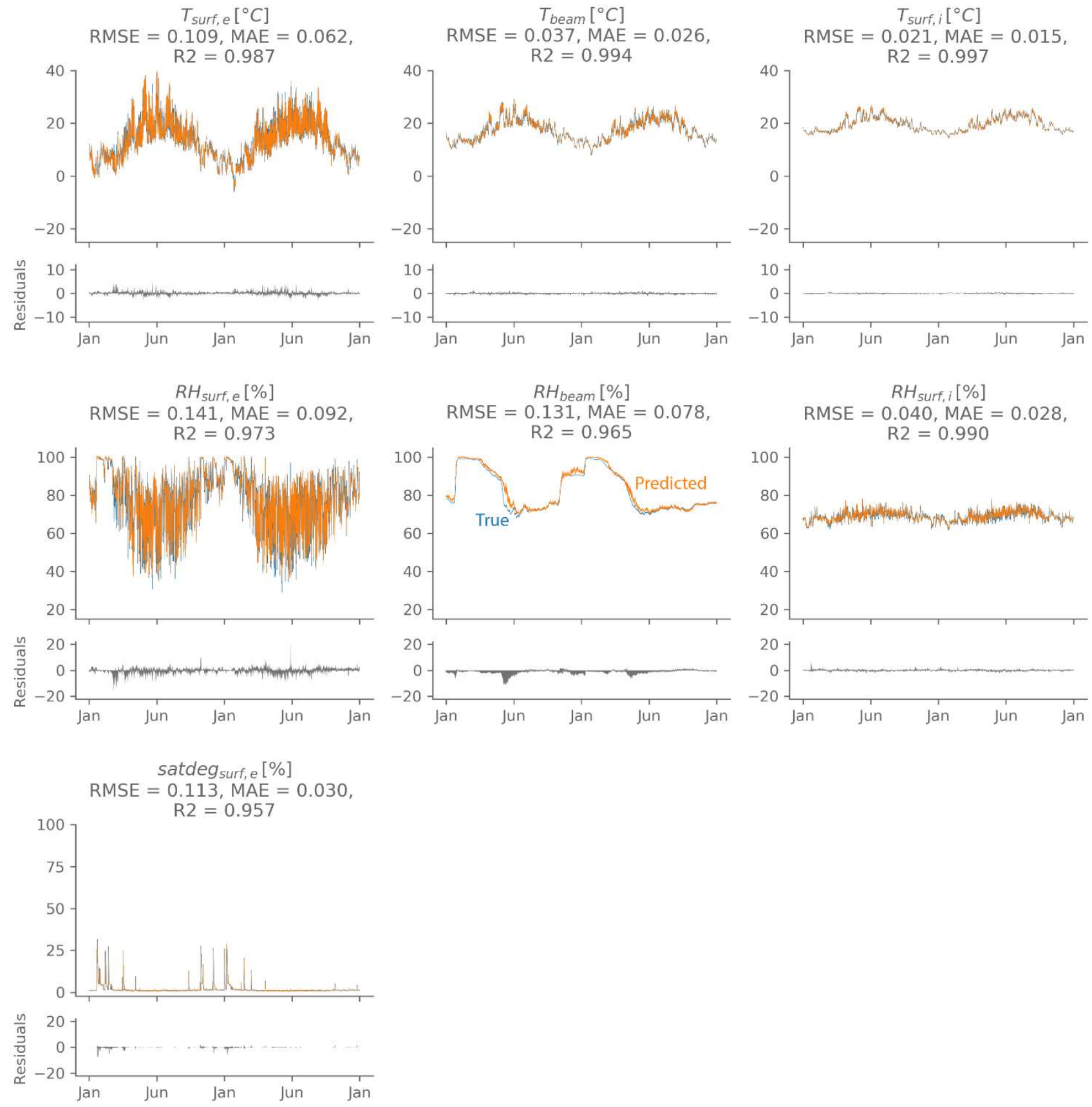

Fig. 3. The hygrothermal predictions for a Belgian climate sample, using CNN-SE-IT.

\section{Results and discussion}

Figure 2 shows the prediction performance on the test sets of both networks, CNN-BE (blue) and CNN-SE-IT (orange). CNN-BE learned the relation between input and output correctly for the Belgian climates, but clearly cannot predict accurately for the Swedish and Italian climates. This means CNN-BE did not learn a mapping that generalises to the Swedish and Italian climates as well. On the other hand, Figure 2 shows that CNN-SE-IT is able to predict accurately for all climates, including the Belgian climates it was not trained on. This is confirmed by Figure 3, which shows the hygrothermal time series for a sample of the Belgian test set, as predicted by CNN-SE-
IT. The predicted value (orange) corresponds very well with the true value (blue), thus indicating good prediction performance. Additionally, Figure 4 shows the accuracy of the damage patterns for the three test datasets, calculated based on the hygrothermal time series as predicted by CNN-BE (top) and CNN-SE-IT (bottom). Again, it is seen that the predictions by CNN-SE-IT (bottom) are accurate for all climates, whereas the predictions by CNN-BE (top) are only accurate for the Belgian climates the network was trained on.

The difference in prediction accuracy of both networks can be explained by looking at the climate data used as input to train and predict. Figure 5 shows the probability distribution functions of the climate parameters and reveals that the range of the Swedish and 

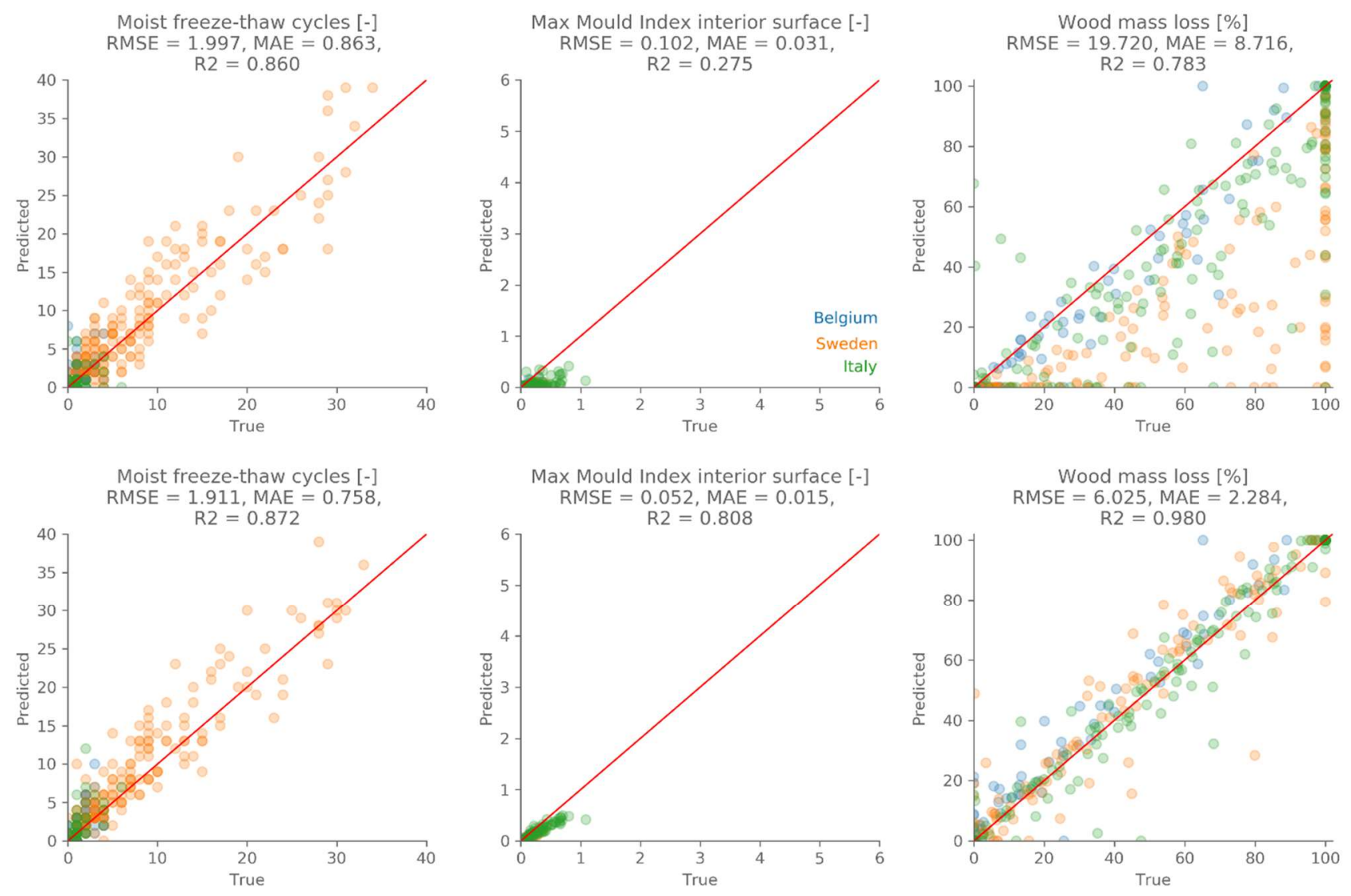

Fig. 4. The prediction accuracy of the damage patterns for the three test datasets shows that CNN-SE-IT (bottom) predicts accurately for all climates, whereas CNN-BE (top) is only accurate for the Belgian climates it was trained on.

Italian climate parameters often exceed the range of the Belgian climate. While neural networks can be trained to map from one specific dataset to another, they usually do not learn a generalized transformation that can extrapolate accurately (far) outside the space of training. Hence, if CNN-BE receives input values that lie (far) outside the range it was trained on (i.e. much lower temperatures or higher rain loads), the network is not able to map this input correctly to output values. On the other hand, the range of the Belgian climates lies perfectly within the range of the Swedish and Italian climates combined. Consequently, CNN-SE-IT is able to map the Belgian climate input to the correct output and predict the hygrothermal time series accurately. This seems to indicate that extrapolation to other climates is possible, under the condition that the range of the new climate data lies within the range of the climates the network was trained on. Thus, while this can be seen as extrapolation from a geographical point-of-view, this is actually interpolation for the neural network.

Finally, the training time of a single CNN is about 45 min on a machine with a dual RTX 2080 8GB GPU. On a machine with 32 processor cores, it took $100 \mathrm{~min}$ to simulate the 768 samples of the training dataset and 35 min to simulate the 256 samples of the validation dataset. Thus, the total development time of the $\mathrm{CNN}$ is 3 hours, during which 1280 samples could be simulated with the hygrothermal model. As the CNN can predict 256 samples in only 20 seconds, it pays off if the probabilistic assessment requires more than 1280 samples.

\section{Conclusion}

In this paper, a convolutional neural network replaces the hygrothermal simulation model for a massive masonry wall, to decrease computational time in a probabilistic framework. The goal was to investigate the possibility to train the network on a dataset with a limited number of exterior climates, and afterwards use the network to predict the hygrothermal performance of the masonry wall in other climates as well. To this extent, the network was trained on two different datasets, one containing four Belgian climates, the other containing four Swedish and four Italian climates. The results showed that the network trained on the latter dataset was able to extrapolate to the Belgian climates, while the network trained on the former dataset could not accurately predict for the Swedish and Italian climates. By analysing the input climate data, it was found that the data range of the Swedish and Italian climates often exceeds the data range of the Belgian climate, whereas the Belgian climate lies perfectly within the range of the Swedish and Italian climates combined. This thus indicates that extrapolation to other climates is possible, as long as the data range of the new climates lies within the range the network was trained on. Hence, from 

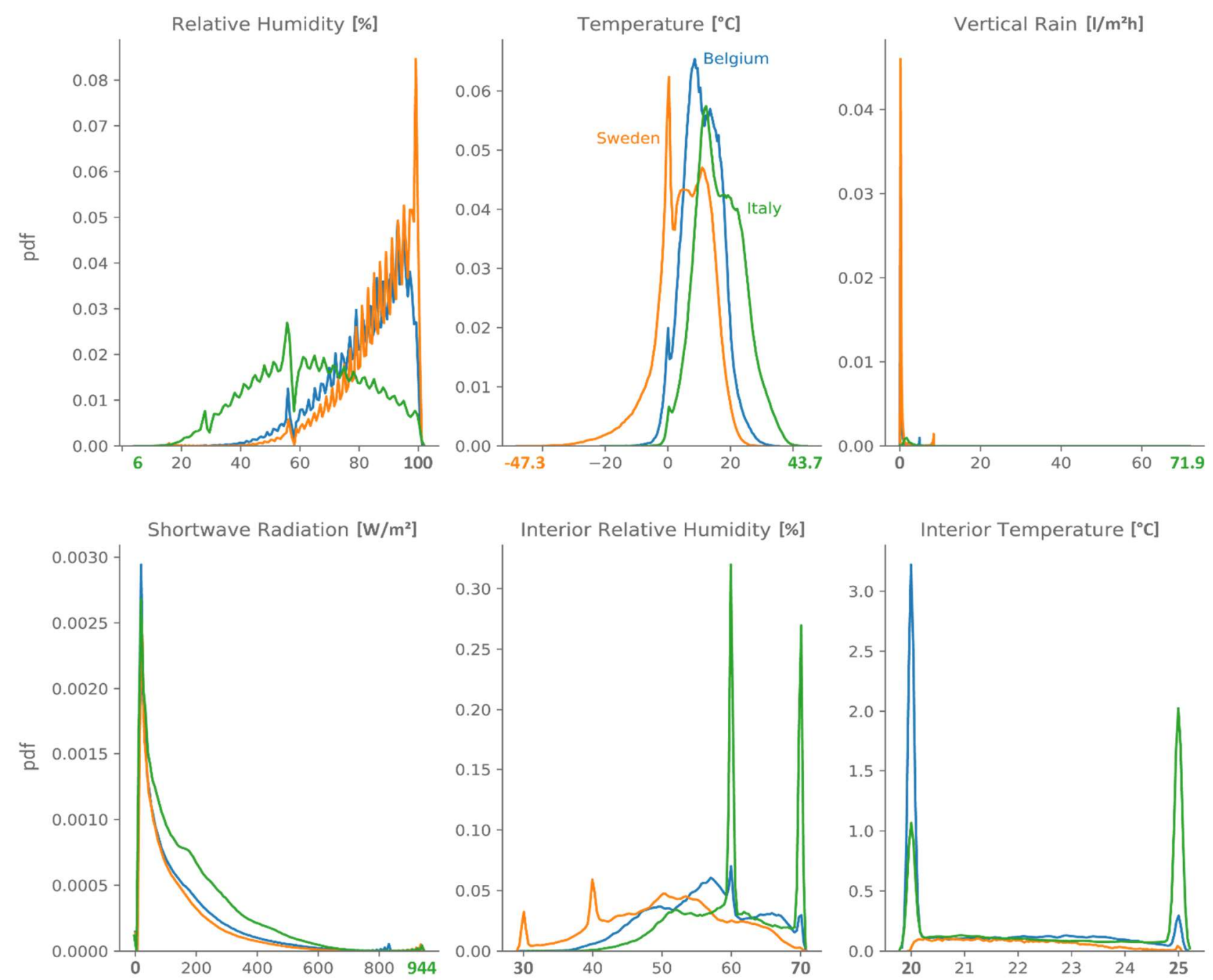

Fig. 5. The probability distribution functions of the climate data used as input for the network explain why the network trained on the Belgian climate (blue) cannot predict for the Swedish (orange) and Italian (green) climates: the input values of these climates lie outside the range of the input data the network was trained on. The minima and maxima are indicated in bold on the x-axis, in the colour of the climate to which it belongs.

the perspective of the network, this is interpolation, rather than extrapolation. Additionally, it was shown that the total computational time can be reduces by employing a convolutional neural network, if the probabilistic assessment requires more than 1280 samples.

This project has received funding from the European Union's Horizon 2020 research and innovation program under grant agreement No 637268. This financial support is gratefully acknowledged.

\section{References}

1. L. Van Gelder, H. Janssen, S. Roels. Energy and Buildings 79, 202-211 (2014)

2. E. Vereecken, L. Van Gelder, H. Janssen, S. Roels. Energy and Buildings 89, 231-244 (2015)

3. H. Janssen. Reliability Engineering \& System Safety 109, 123-132 (2013)

4. A. Tijskens, S. Roels, H. Janssen. Building and Environment 162, 106282 (2019)
5. A. Tijskens, H. Janssen. Energies 12, 3966 (2019)

6. E. Vereecken, S. Roels. Construction and Building Materials 41, 697-707 (2013)

7. Climate For Culture, European Commission. (2014)

8. B. Blocken, J. Carmeliet. Wind and Structures, An International Journal 5, 441-462 (2002)

9. European committee for Standardisation. (2007)

10. T. Hou, D. Nuyens, S. Roels, H. Janssen. Reliability Engineering and System Safety, Elsevier, vol. 191 (2019) 